\title{
UGUAGLIANZA E DIFFERENZA DI GENERE NELLA RIFORMA DEL DIRITTO DI FAMIGLIA: UNA LETTURA LUHMANNIANA
}

\author{
Stefano MAGNOLO \\ Professore Aggregato di Sociologia del Diritto \\ Dipartimento di Scienze Giuridiche \\ Università di Salento \\ stefano.magnolo@unisalento.it
}

\begin{abstract}
RESUMEN
La igualdad ante la ley es uno de los derechos fundamentales de las Constituciones modernas. Sin embargo, hay algo más. De hecho podría decirse que los modernos sistemas jurídicos se han ido formando alrededor de la idea de igualdad. El valor de la igualdad parece expresar una manera común de entender las relaciones bumanas, especialmente en el caso de la igualdad de género, expresamente mencionado por el artículo de las Constituciones democráticas modernas que se refiere al principio de igualdad ante la ley. El presente estudio trata esa forma especifica de igualdad que se caracteriza por la paradoja de querer tratar como igual lo que es diferente, o sea, de hacer distinciones sin discriminar. Con este fin nos referimos al enfoque jurídico y al enfoque político del tema como se encuentran en la reforma del derecho de familia. No es un problema de una específica legislación nacional, sino más bien una versión especifica de un problema de nivel general. Se trata de la imposibilidad de construir como indistinguible lo que es diferente. El intento politico de reformar el derecho de familia para implementar el principio constitucional de igualdad no consigue resolver el problema. Éste vuelve a aparecer por la imposibilidad que el derecho funcione sin crear diferencias. Al mismo tiempo, surgen problemas nuevos relacionado con formas nuevas de organizar la vida familiar.
\end{abstract}

Palabras clave: igualdad, matrimonio, amor, relaciones humanas, sociología Luhmann.

\section{ABSTRACT}

Even if equality before the law is a basic buman right in modern constitutions there is much more to that. In fact, we could say that legal systems have arisen around the idea of equality. The full meaning of equality seems to express a common way to unsderstand buman relationships, particularly in the case of gender equality, which is specifically mentioned by modern democratic constitutions and which relates to the principle of equality before the law. The current paper approaches such specific way of equality whose features are the paradox of equal treatment of the unequal, that is to say, distinction without discrimination. To that purpose we refer to the legal and political approach to the subject as it is 
Stefano Magnolo Uguaglianza e differenza di genere nella riforma del Diritto...

seen in the reform of Family Law. This is not a matter of a given country's legal system, but a particular version of a general matter, which is the impossibility to see as indistinguible which is actually different. Political attemps to reform Family Law to implement the constitutional law of equality does not solve the issue. A matter as such appears once again at the impossibility of the law to work without establishing differences. At the same time, new problems related to new ways to organise family life appear.

Keywords: Equality, marriage, love, human relationships, Sociology according to Luhmann.

\section{ZUSAMMENFASSUNG}

Die Gleichbeit vor dem Gesetz ist eines der Grundrechte moderner Verfassungen. Jedoch kommt noch etwas hinzu. Man könnte sagen, dass die modernen Rechtssysteme um das Gleichbeitsprinzip herum entstanden sind. Der Wert der Gleichbeit scheint im Verständnis der menschlichen Beziebungen eine Gemeinsamkeit darzustellen, insbesondere im Fall der Geschlechtergleichbeit, so wie dies von den modernen demokratischen Verfassungen in einem eigenen Paragraphen festgebalten wird, der sich auf die Geschlechtergleichbeit vor dem Gesetz beziebt. Die vorliegende Studie beschäftigt sich mit dieser besonderen Form der Gleichheit, die durch das Paradox charakterisiert ist, als gleich zu behandeln, was verschieden ist bzw. den Unterschieden gerecht werden zu müssen, obne zu diskriminieren. Zu diesem Zweck beziehen wir uns auf die juristische und die politische Perspektive des Themas, so wie sie in die Reform des Familienrechts Eingang gefunden hat. Dies ist kein Problem einer nationalspezifischen Rechtsgebung, sondern eher eine nationalspezifische Version eines allgemeinen Problems. Es gebt um die Unmöglichkeit, das als ununterscheidbar zu konstruieren, was verschieden ist. Der politische Versuch der Reform des Familienrechts um das Verfassungsprinzip der Gleichbeit zu implementieren vermag dieses Problem nicht zu lösen. Dieses taucht wieder auf, und zwar bedingt durch die Unmöglichkeit des Gesetzes wirksam zu sein, obne Unterschiede zu schaffen. Zur gleichen Zeit entstehen neue Probleme im Zusammenhang von neuen Formen der Organisation familiären Lebens.

Schlüsselwörter: Gleichheit, Ehe, Liebe, menschliche Beziehungen, Soziologie Luhmann.

SUMARIO: I. INTRODUZIONE.-II. LA FAMIGLIA E IL DIRITTO PUBBLICO E PRIVATO.-III. LA FAMIGLIA E LA SOCIOLOGIA.-IV. COSTITUZIONE, UGUAGLIANZA E STRUTTURA SOCIALE.-V. L'INDIVIDUO E LA FAMIGLIA._-VI. LA FAMIGLIA, IL MATRIMONIO E L'AMORE.VII. DIRITTO E POLITICA: LA RIFORMA E LA COSTITUZIONE.VIII. UGUAGLIANZA, STRUTTURA E TEMPO.-IX. DIRITTI UGUALI PER SOGGETTI UGUALI?-X. FAMIGLIE SENZA MODELLI.-XI. MATRIMONIO DI GENERE?-XII. DONNE E UOMINI. 


\section{INTRODUZIONE}

Il tema dell'uguaglianza rappresenta uno dei temi cardine del discorso intorno alla realizzazione dei presupposti di ciò che è stato definito come il progetto moderno. Non si tratta, ovviamente, di una questione esclusivamente moderna. La riflessione filosofica ha da sempre esaltato il valore dell'uguaglianza come espressione della giustizia sin dalle rappresentazioni cosmologiche dei filosofi presocratici. Tuttavia, a partire dalla sua costituzionalizzazione, il discorso sull'uguaglianza assume caratteri specificamente moderni, in quanto con essa si riconosce l'esistenza di disuguaglianze sociali e personali e si afferma l'irrilevanza giuridica di queste, quando non la necessità del loro superamento. La vicenda delle codificazioni moderne si presta, sotto questo aspetto, a varie letture che esaltano il riconoscimento del valore dell'uguaglianza come realizzazione degli ideali rivoluzionari tesi all'abbattimento di vecchie gerarchie e privilegi. Su queste trasformazioni, la teoria dei sistemi consente di articolare una sostanziale convergenza verso il tema dell'inclusione sia per la questione dell'uguaglianza, sia per la funzione della famiglia. Con il passaggio alla modernità, infatti, la forma primaria della differenziazione rompe con le disuguaglianze di rango e le gerarchie della stratificazione orientandosi all'uguaglianza sia nel senso della eterarchia dei sottosistemi, sia come realizzazione di un nuovo principio di inclusione. In questo modo è data, allo stesso tempo, la trasformazione della famiglia, che non costituisce più quell'unità multifunzionale indispensabile per l'inclusione nei sottosistemi delle società premoderne e per la costruzione di un ordine che si basava su differenti collocazioni sociali articolate in base all'appartenenza familiare. Astrazione del soggetto e individualizzazione della persona, accanto alla pluralità dei ruoli dell'individuo e alla crescente separazione tra relazioni personali e impersonali, fanno da sfondo alla realizzazione della possibilità di una inclusione universale nei nuovi ambiti della comunicazione, giuridicamente riconosciuta con il principio costituzionale di uguaglianza, e il bisogno della piena inclusione della persona, che emerge come possibilità offerta solo dalla famiglia, mentre è indipendente dalle altre inclusioni legate a ruoli specifici dell'individuo.

Si tratta di trasformazioni che, dal punto di vista strutturale, si sostanziano nella formazione di nuovi sistemi grazie ad acquisizioni evolutive che rendono possibile l'autonomia funzionale della famiglia e la sua strutturazione in funzione della istituzionalizzazione dell'amore come fondamento del matrimonio. 
Una nuova rilevanza che lo stato moderno riconosce attraverso la particolare tutela accordata alla famiglia e al matrimonio. La regolazione giuridica di questo ambito nell'ordinamento italiano si esprime non solo con le norme costituzionali specificamente dedicata alla famiglia (artt. 29, 30 e 31; tutti nel titolo II della Costituzione, dedicato ai rapporti etico-sociali) ma anche con il diritto fondamentale di uguaglianza, del resto alla base della formulazione dell'art. 29, e con le norme del codice civile che formano il diritto di famiglia. Quest'ultimo, d'altra parte, si espone a quella mutabilità strutturale tipica del diritto nella forma della legislazione ordinaria, che invece non è propria delle norme costituzionali, e attraverso la quale si esprimerebbe la co-evoluzione tra diritto e società. Inoltre, va detto che, in relazione ai mutamenti sociali, secondo alcuni la famiglia avrebbe una limitata capacità di adattamento, tenuto conto della sua «dipendenza» dalla evoluzione della specie umana; e secondo altri, al contrario, sarebbe dotata di estrema adattabilità, se si guarda ai rapidi mutamenti di costume. Se teniamo conto anche di questo, i modelli di regolazione giuridica della famiglia sembrano prestarsi all'analisi del rapporto tra diritto e mutamento sociale ${ }^{1}$. In generale, porre la questione del diritto costituzionale di uguaglianza nel caso del divieto di discriminazioni di genere non lo caratterizza diversamente rispetto al generico divieto di distinzioni irragionevoli nella interpretazione che si fa del contenuto dell'art. 3. Tuttavia, la sua contestualizzazione nel diritto di famiglia fa assumere alla questione connotati particolari che speriamo si chiariscano alla fine del nostro discorso. Qui, intanto, è interessante notare come sulla questione dell'uguaglianza tra uomo e donna si sia prodotta una tensione costante tra diritto di famiglia e costituzione, al di là delle questioni concernenti il riconoscimento giuridico delle unioni non coniugali. Diversa, invece, in relazione al principio di uguaglianza, come facilmente intuibile, la rilevanza del dibattito in merito alle unioni ed ai matrimoni tra persone dello stesso sesso, questione che deve confrontarsi con un diritto di famiglia che in fondo deve basarsi sulla differenza di genere, ma che da questa, in osservanza al principio di uguaglianza, non può far dipendere «irragionevoli» distinzioni.

La nostra argomentazione tenterà allora di rispondere alle questioni: è possibile una politica di effettiva uguaglianza di genere? Che conseguenze ha nel diritto di famiglia il perseguimento della uguaglianza tra uomo e donna?

${ }^{1}$ Cfr. R. KÖNIG, «Diritto», in ÍD. (a cura di), Sociologia, Milano, Feltrinelli, 1964, pp. 94-95, e V. FERRARI, Lineamenti di sociologia del diritto, vol. I, Azione giuridica e sistema normativo, Roma-Bari, Laterza, 1999. 


\section{LA FAMIGLIA E IL DIRITTO PUBBLICO E PRIVATO}

Nonostante le notevoli differenze di carattere teorico e metodologico tra sociologia della famiglia e diritto di famiglia, è possibile rintracciare una sovrapposizione di forme della rappresentazione sociale fra le due discipline. Almeno fino alla riforma del 1975, il diritto moderno usa la retorica del buon padre di famiglia, e pone a capo della famiglia il maschio procacciatore di risorse. In maniera analoga, Talcott Parsons, nella biografia normale dell'uomo qualunque si riferisce al maschio come breadwinner:

«Supponiamo di avere un individuo in cui il modello generale di orientamento di valore della realizzazione universalistica, della specificità, della neutralità e dell'orientamento in vista della collettività sia ben stabilito nella struttura della sua personalità fondamentale. In primo luogo, essendo un maschio, egli deve apprendere che da un uomo, una volta "cresciuto" in maniera da ricoprire un ruolo professionale, ci si attende che "abbia un posto", che "si guadagni il pane", e molto probabilmente che mantenga una famiglia. Egli deve apprendere che il sistema professionale è ordinato gerarchicamente e che, se egli ha una spiccata ambizione al successo, deve cercare di raggiungere uno dei livelli più elevati del sistema professionale»².

Prima della riforma del 1975, in Italia la famiglia era regolata dalla codificazione del 1942. Si tratta di una regolazione che in parte si ispirava ancora al modello della codificazione napoleonica che permeava i sistemi giuridici continentali. Diversi gli aspetti interessanti di questa regolazione, che da un lato sembra contraddire il carattere individualistico del code, poiché subordina le libertà individuali all'interesse superiore della istituzione familiare. Dall'altro lato, in modo sostanzialmente convergente con i sistemi di common law di ispirazione vittoriana, il riconoscimento di distinte identità giuridiche per i componenti della famiglia è inserito all'interno della giuridicizzazione delle relazioni in senso fortemente gerarchico. Un altro aspetto apparentemente contraddittorio è costituito dal fatto che famiglia e matrimonio esprimono la concezione dei rapporti derivanti dalla separazione tra stato e società, una separazione che è stata alla base della interpretazione dogmatica della funzione dei diritti fondamentali. Tuttavia, nonostante questa separazione, la sfera privata della famiglia è modellata sulla sfera pubblica dello stato, poiché, in quanto considerata come fon-

2 T. PARsons, Il sistema sociale, Milano, Comunità, 1996, p. 247. 
damento della società civile, assume una funzione di rilevanza politica. Lo stesso accade nel common law, dove, a fronte del riconoscimento del carattere privato della famiglia, il matrimonio non ha però natura squisitamente privatistica ${ }^{3}$.

Il matrimonio, inoltre, sembra essere il bersaglio privilegiato dei controlli istituzionali e sociali tesi a limitare la libertà individuale; così la sua regolazione nel code costituisce la reazione dello stato al controllo operato dalla Chiesa. Stato che si sostituisce a quest'ultima. Così descrive Chiara Saraceno questo aspetto: «Il matrimonio, contratto tra individui liberi, è innanzitutto un contratto patrimoniale, che fonda una precisa gerarchia tra i sessi, in nome, appunto, della unità patrimoniale della nuova famiglia, sulla base del nuovo valore attribuito all'individuo - maschio, possidente.[...] Società di individui-cittadini, la società borghese è innanzitutto società di capifamiglia, ove i maschi sono tendenzialmente svincolati dalla tutela parentale [...], ma le femmine possono esercitare formalmente la propria libertà solo nell'atto del passaggio da una tutela (del padre) ad un'altra (del marito)» ${ }^{4}$.

Questi aspetti descrivono quella che è stata definita come «la doppia anima del codice napoleonico». In quella codificazione e nella successiva regolamentazione della famiglia convergono la separazione dello stato dalla società, ma anche la crisi della economia domestica e lo sviluppo della economia monetaria separata dalla politica. Assunti individualistici ed egualitari convivono con relazioni di tipo gerarchico, tipiche della struttura dello stato, che si riflette in parte sulla sfera privata, anche se il potere del capofamiglia inizia ad essere limitato ${ }^{5}$. La riflessione teorica vede ormai consolidarsi la separazione tra stato e società. Tra la sfera privata della famiglia, che non costituisce più un elemento fondamentale dello stato, e la sfera pubblica, si collocherebbe la società civile, poiché quest'ultima non si esaurisce nei soli rapporti economici ${ }^{6}$. Così, la regolamentazione di quella sfera «riguarda la stessa organizzazione della società borghese, la sua peculiare distinzione tra sfera pubblica e sfera privata, tra responsabilità pubbliche e responsabilità private, che segna tuttora profondamente il modo in cui pensiamo sia alla famiglia e al rapporto uomo-donna, che al rapporto tra la famiglia e la società e lo stato» ${ }^{7}$.

Cfr. V. Pocar e P. Ronfani, La famiglia e il diritto, Roma-Bari, Laterza, 2003, pp. 3-13.

${ }^{4}$ C. Saraceno, Sociologia della famiglia, Bologna, Il Mulino, 1996, p. 95.

${ }^{5}$ Cfr. ivi, pp. 92 e 177.

${ }^{6}$ Cfr. N. Matteucci, Lo stato moderno, Bologna, Il Mulino, 1993, pp. 39-45.

7 C. Saraceno, Sociologia della famiglia, op. cit., p. 95. 


\section{LA FAMIGLIA E LA SOCIOLOGIA}

Il riferimento alla funzione della famiglia trova collocazione in relazione a quelle trasformazioni legate all'affermazione della struttura sociale moderna e che hanno portato a tematizzare una «perdita di funzioni della famiglia» rispetto a strutture sociali storicamente precedenti. Ci troviamo, a questo punto, ad uno stadio relativamente avanzato della riflessione propriamente sociologica sulla famiglia. Una riflessione che si afferma, infatti, solo a partire dalla metà del XIX secolo e che solo con lo struttural-funzionalismo raggiungerà uno statuto epistemologico esente da giudizi di valore. Già in Durkheim, in senso evoluzionistico, troviamo il riferimento ad una «"contrazione" della famiglia, sino al gruppo minimo della famiglia coniugale» come una «legge generale dello sviluppo della famiglia» ${ }^{8}$. Successivamente questa «perdita di funzioni» entrerà nelle acquisizioni stabili delle discipline sociologiche grazie al testo di Talcott Parsons e Robert Bales, tradotto in italiano con il titolo di Famiglia e socializzazione ${ }^{9}$. Nella moderna sociologia della famiglia, puntualizza Luhmann, «ist wobl allgemein akzeptiert, daß "Funktionsverlust" nicht einfach Abnahme der gesellschaftlichen Bedeutung der Familie besagen kann, sondern auf funktionale Spezifikation binausläuft mit Entlastungen auf der einen Seite und Intensivierungen auf der anderen» ${ }^{10}$. In Parsons questa perdita viene appunto chiarita con riferimento al ruolo della famiglia nella società moderna, ruolo che sembra essere in crisi. Questa crisi dipenderebbe dal fatto che la famiglia attraversa una fase di transizione da unità «polifunzionale» a unità che svolge funzioni specifiche: la socializzazione dei figli e la definizione della personalità degli adulti ${ }^{11}$. Ai nostri fini interessa qui rilevare come la posizione struttural-funzionalista in merito alla famiglia tenga conto della struttura complessiva della società, dove la rilevanza della famiglia si definisce come struttura differenziata, le cui funzioni presuppongo-

${ }^{8}$ R. KÖNIG, «Famiglia», in ÍD. (a cura di), Sociologia, op. cit., p. 145. Del resto, aggiunge l'autore alla stessa pagina, «le oscillazioni nella concezione moderna dell'individualismo rispecchiano [...] chiaramente le circostanze esterne in cui si è sviluppata la sociologia della famiglia».

9 T. Parsons e R. F. Bales, Famiglia e socializzazione, Milano, Mondadori, 1974.

${ }^{10}$ Cfr. N. Luhmann, «Sozialsystem Familie», in íD., Soziologische Aufklärung, vol. V, Wiesbaden, VS Verlag, 2005, pp. 189-209 (198). D’altra parte, Luhmann non manca di evidenziare come, nonostante questa perdita di funzioni, alla famiglia vengano ancora attribuite una pluralità di funzioni.

${ }_{11}$ T. Parsons e R. F. BALes, Famiglia e socializzazione, op. cit., pp. 22-31. 
no la differenziazione di ruoli di genere. Al tempo stesso la specificazione funzionale in questo senso attesta la perdita di una seria di altre funzioni che le erano proprie e, attraverso il legame coniugale, definisce i propri confini rispetto agli altri sistemi della società. Tuttavia, nonostante questa differenziazione della famiglia come luogo, privato, delle relazioni basate sull'affettività, vi è, forte, una connessione tra i ruoli interni alla famiglia, differenziati in base all'età e soprattutto al sesso, ed altri ruoli «esterni» socialmente rilevanti, di modo che il rispetto della differenziazione dei ruoli familiari è funzionale alla stabilità dell'intera struttura sociale. Ciò ha due conseguenze, da un lato la connessione pubblico-privato e, dal punto di vista del diritto, il fatto che le norme sulla famiglia costituiscono solo in apparenza la regolazione di una sfera privata. Vedremo come questa connessione pubblico-privato compaia nel diritto di famiglia e come, attraverso questa connessione, ricompaia nel diritto la questione della differenza di genere. In ogni caso, nella rappresentazione di Parsons e Bales, la famiglia, nonostante la «perdita di funzioni», e grazie alla sua specializzazione risulta avere una rilevanza fondamentale per la società. Da qui, potremmo dire, l'attenzione del diritto per questo ambito.

Il quadro cambia se, riprendendo l'affermazione di Luhmann relativa alla perdita di funzioni della famiglia, osserviamo la sua descrizione della funzione della famiglia nella struttura della società moderna. Lo facciamo a partire dal testo del 1965, Grundrechte als Institution. Il riferimento a questo testo di Luhmann è importante per vari motivi. Un primo è rappresentato dal fatto che, come afferma il sottotitolo, Ein Beitrag zur politischen Soziologie, si tratta del primo tentativo di affrontare dal punto di vista sociologico un tema fino a quegli anni monopolio della dogmatica giuridica. A tal fine presenta, collegandoli fra loro, gli aspetti che vogliamo trattare inserendoli nell'ambito di una concezione complessiva della struttura sociale moderna. Seppure con una architettura teorica ancora acerba, infatti, Luhmann descrive la funzione della famiglia, del principio di uguaglianza, il funzionamento del medium dell'amore. Al principio di uguaglianza davanti alla legge è dedicato un intero capitolo, mentre la questione relativa alla tutela del matrimonio e della famiglia sono trattate nel capitolo che Luhmann dedica alla «civilizzazione delle aspettative di comportamento: libertà di comunicazione». Descrivendo l' «alto livello di mobilità dei contattii come «uno dei più importanti fenomeni degli ordini sociali differenziati», Luhmann ne ravvede una conseguenza nel principio, anch'esso dunque tipicamente moderno, della istituzionalizzazione dell'amore come fondamento del matrimonio. In questo senso, potremmo 
dire, l'amore diventa nella società moderna condizione necessaria e sufficiente per la scelta del partner, senza che sia necessario alcun controllo sociale da altri ruoli esterni, o anche relativi alla famiglia. La tutela di una libera scelta, tuttavia, sarebbe garantita da altri istituti giuridici ${ }^{12}$. Sinteticamente, riguardo alla funzione della famiglia, ritroviamo la tesi della «perdita di funzioni» della famiglia moderna rispetto al passato, dovuta alla trasformazione strutturale della società. Con la differenziazione sociale moderna si assisterebbe ad una specificazione funzionale della famiglia nella «socializzazione del bambino» e come «spazio per l'autorappresentazione personale». Quest'ultimo aspetto è rilevante in quanto la molteplicità dei ruoli che rivestono i singoli negli ambiti della differenziazione della società moderna, ruoli che a loro volta non sono più connessi con la famiglia e neanche necessariamente connessi fra loro, non offre sufficienti occasioni per un comportamento che non sia orientato ad essi, deviante rispetto a ciò che i ruoli prescrivono. La famiglia, allora, sarebbe l'unico «luogo» della società moderna dove il singolo può rappresentare una sintesi personale di tutti i ruoli. Sono queste due funzioni, secondo Luhmann, a trovare protezione nei confronti della sfera politica con un diritto fondamentale corrispondente. Qui si chiarisce la sua scelta di trattare la tutela costituzionale del matrimonio e della famiglia tra i diritti fondamentali relativi alla libertà di scelta dei temi e dei partner della comunicazione. D'altra parte, invece, la strutturazione della famiglia, e in particolare la questione della equiparazione tra uomo e donna (qui in connessione l'art. 6 e l'art. 3, II comma della Costituzione tedesca), non dovrebbe rientrare tra le questioni di rango costituzionale, sovraccaricando la politica ed il diritto. In questo modo, infatti, si ottiene l'effetto di trasformare la famiglia in un tema politico, oggetto di pianificazione e di programmi elettorali ${ }^{13}$.

${ }^{12}$ Curiosamente, ma efficacemente, Luhmann, nel paragonare questa caratteristica degli ordini differenziati moderni al funzionamento del libero mercato, evidenzia come «Nur weil das politsche Eingriffsinteresse bei Eheschließungen geringer ist als bei wirtschaftlichen Verfugüngen, kennen unsere Verfassungen kein Menschenrecht auf Liebesheirat» [N. LuHMANN, Grundrechte als Institution, Berlin, Duncker \& Humblot, 1965, pp. 88-90 (90)]. Il riconoscimento della protezione costituzionale alla famiglia ed al matrimonio dell'art. 6, I comma della Costituzione tedesca: «Il matrimonio e la famiglia godono della particolare protezione dell'ordinamento statale», non stabilisce, in ogni caso, un riconoscimento del diritto fondamentale al matrimonio d'amore, ma un rafforzamento della garanzia giuridica della libertà di scelta del partner.

${ }_{13}$ Cfr. ivi, pp. 98-107. Queste libertà, chiarisce Luhmann: «Sind mit jenen Errungeschaften, die wir als Zivilisation der Erwartungen bezeichnet haben, auf vielfältige, zum Teil sebr indiskrete und schwer durchschaubare Weise verbunden. Sie dienen dadurch der Lösung des Problems, von dem wir in diesem Kapitel ausgingen: wie in stark differenzierten Sozialordnun- 
A questo punto abbiamo gli elementi per far convergere il discorso del principio costituzionale di uguaglianza nell'ambito della struttura sociale moderna con il diritto moderno. Successivamente ci occuperemo dei problematiche attuali della famiglia e del diritto di famiglia.

\section{COSTITUZIONE, UGUAGLIANZA E STRUTTURA SOCIALE}

Il giudizio di uguaglianza presuppone la diversità di ciò che si confronta $^{14}$. Per quanto possa sembrare paradossale, questa constatazione è alla base dell'articolazione del giudizio di legittimità costituzionale di una norma rispetto all'art. 3 della Costituzione italiana ${ }^{15}$. Tale giudizio, infatti, è l'unico a non svolgersi secondo uno schema binario come tutti gli altri, poiché prevede un confronto della norma in esame con un tertium comparationis, oltre che con l'art. 3 della Costituzione ${ }^{16}$. Alla base dell'applicabilità del principio costituzionale di uguaglianza vi sono fondamenti logici che discendono dalla sua formulazione. Anche come principio giuridico, allora, l'uguaglianza sembra a Kelsen ${ }^{17}$ vuota di contenuto. Il suo esame delle formule della giustizia lo porta ad affermare una sostanziale coincidenza della struttura logica delle esplicitazioni dell'uguaglianza nei moderni ordinamenti democratici con quelle formule storicamente precedenti. Si tratta di una struttura che non fornisce alcun criterio operativo e che funziona solo sulla base della presupposizione ontologica di una determinata struttura sociale. La «vuotezza» delle formulazioni riguardanti valori assoluti è la stessa per le norme

gen bei entsprechend individualisierten Selbstdarstellungsinteressen ausreichende Komplementarität der Verhaltenserwartungen sichergestellt werden kann» (ivi, p. 100).

${ }^{14}$ Cfr O. Dann, «Gleichheit», in O. Brunner, W. Conze e R. Koselleck (a cura di), Geschichtliche Grundbegriffe. Historisches Lexikon zur politisch-sozialen Sprache in Deutschland, vol. 2, Stuttgart, Klett-Cotta, 1979, pp. 997-1046.

15 «Tutti i cittadini hanno pari dignità sociale e sono uguali davanti alla legge senza distinzione di sesso, razza, lingua, religione, opinioni politiche, condizioni personali e sociali» (art. 3, I comma). «È compito della Repubblica rimuovere gli ostacoli di ordine economico e sociale che, limitando di fatto la libertà e l'eguaglianza dei cittadini, impediscono il pieno sviluppo della persona umana e l'effettiva partecipazione di tutti i lavoratori all'organizzazione politica, economica e sociale del Paese» (art. 3, II comma).

${ }^{16}$ Cfr., fra gli altri, C. Rossano, L'eguaglianza giuridica nell'ordinamento costituzionale, Napoli, E. Jovene, 1966.

${ }_{17}$ Cfr. H. KeLsen, «Che cos'è la giustizia», in ÍD., I fondamenti della democrazia, Bologna, Il Mulino, 1970, pp. 393-433; cfr. anche íD., Il problema della giustizia, Torino, Einaudi, 1975. In questo senso anche A. PODLECH, Gehalt und Funktionen des allgemeinen verfassungsrechtlichen Gleichbeitssatzes, Berlin, Duncker \& Humblot, 1971, che parla di svuotamento semantico. 
aventi valore generale, come nel caso delle formule «la legge è uguale per tutti» e «tutti sono uguali davanti alla legge». In particolare, il principio di uguaglianza, nella concezione kelseniana, sarebbe accostabile alla formula dell' «uguale all'uguale», il Gleiches zu Gleichem ${ }^{18}$, che si sviluppa nelle idee platoniche e aristoteliche della uguaglianza geometrica e della giustizia proporzionale accolte nella successiva formulazione del suum cuique.

Queste formulazioni hanno una valore operativo solo sulla base del presupposto di una struttura di disuguaglianze che predetermina ciò che spetta a ciascuno. Ed è solo in questo contesto, come afferma Luhmann, che la formula del suum cuique può avere senso «weil das einem Menschen Zustehende nicht für alle dasselbe ist ${ }^{19}$. Il fatto che ciò che spetta non sia uguale è però un presupposto, è dato dal fatto naturale della disuguaglianza, reso evidente da un ordine sociale differenziato gerarchicamente il quale, attraverso questa formulazione della giustizia, viene confermato $^{20}$. In questo senso, la formula aristotelica del giusto mezzo, come anche il principio del suum cuique, sono «una tautologia dietro la quale si nasconde in varia forma e in laborioso travisamento il principio logico dell'identità, il giudizio che il buono è buono e non cattivo, che il giusto è giusto e non ingiusto, che $a$ è uguale ad $a$ e non è non $a »^{21}$.

Anche Podlech, rispetto alle formulazioni del principio di uguaglianza nella Costituzione tedesca, si chiede quale possa essere il senso della previsione dell'art. 3 e se sia corretta l'interpretazione nel senso di trattare l'uguale in modo uguale e il disuguale in modo disuguale che corrisponderebbe all'espressione della giustizia. Anche per lui, in questo caso, si tratta di una tautologia, che non può funzionare da sola senza ulteriori indicazioni. La sua conclusione, allora, in riferimento alla interpretazione del principio di uguaglianza dell'art. 3, comma I (Alle Menschen sind vor dem Gesetz gleich) è che questo abbia, come anticipato alla nota 17 , contenuto pragmatico e non semantico, rintracciabile in una, di volta in volta, specifica «Argumentationslastregel». Così, i commi II e III dello stesso articolo non contengono norme giuridiche, ma descrivono criteri negativi per l'ammissibilità di un trattamento diseguale ${ }^{22}$.

18 Cfr. C. W. MüLleR, Gleiches zu Gleichem. Ein Prinzip frühgriechischen Denkens, Wiesbaden, O. Harrassowitz, 1965.

${ }_{19}$ N. Luhmann, Das Recht der Gesellschaft, Frankfurt am Main, Suhrkamp, 1995, p. 224.

${ }^{20}$ Cfr. N. Luhmann, «Über Natur», in ÍD., Gesellschaftsstruktur und Semantik: Studien zur Wissenssoziologie der modernen Gesellschaft, vol. IV, Frankfurt am Main, Suhrkamp, 1999, pp. 9-30.

${ }_{21}$ H. Kelsen, Lineamenti di dottrina pura del diritto, Torino, Einaudi, 1998, pp. 56-59.

${ }^{22}$ Cfr. A. PoDlech, Gehalt und Funktionen des allgemeinen verfassungsrechtlichen Glei- 
Se il diritto moderno nega le disuguaglianze esistenti, se il punto di partenza è l'uguaglianza e non una struttura di disuguaglianze, il diritto deve poter distinguere tra uguaglianza e disuguaglianza per poter operare. A tal fine, deve determinare dei criteri che consentono di distinguere e che non essendo presupposti, non solo devono essere rinvenuti successivamente, ma devono essere creati. E questo ogniqualvolta la regola della giustizia come formula di contingenza del sistema giuridico non consente la corretta applicazione dei valori del codice ragione/torto. Vale a dire, ogni volta che si presenta un caso nuovo, non riconducibile alla regola di decidere casi uguali in modo uguale e casi disuguali in modo disuguale, ma anche nella scelta del punto di vista rilevante per il confronto ${ }^{23}$.

D'altra parte, anche in ambito giuridico si tratta di criteri contingenti, non obiettivamente determinabili dal legislatore o dal giudice, ma che una volta formulati sono vincolanti, poiché contenuti in una disposizione normativa: «non si tratta più di un comune giudizio di uguaglianza, in cui si afferma ad esempio che due oggetti A e B sono eguali per determinate caratteristiche, ma di una statuizione giuridica che impone che due oggetti A e B sono e devono essere eguali per determinate caratteristiche, con conseguenze giuridiche particolari a tale eguaglianza connesse» ${ }^{24}$. In questo modo, l'applicazione del principio di uguaglianza se da una parte nega le disuguaglianze sociali, dall'altra deve ammettere la possibilità di disuguaglianze create dal diritto. Lo spazio di contingenza aperto dall'uguaglianza deve essere riempito.

\section{L'INDIVIDUO E LA FAMIGLIA}

Il cambiamento strutturale verso la modernità e la connessa questione della identità e della individualità possono essere descritti anche dal punto di vista dei ruoli dell'individuo. Il riferimento ai ruoli, inoltre, ci riporta al discorso sui diritti fondamentali e sulla tematizzazione dell'uguaglianza. Alla concretezza della nascita come principio di inclusione infatti ora si

chheitssatzes, op. cit., pp. 90-91. Distinzioni nelle fattispecie del II comma (genere) e discriminazioni positive o negative basate sulle fattispecie del III comma (sesso, nascita, razza, lingua, nazionalità o provenienza, fede, opinioni religiose o politiche) non costituiscono mai un «motivo sufficiente per un trattamento diseguale» (ein zureichender Grund für Ungleichbebandlungen).

${ }^{23}$ Sulla giustizia come «Kontingenzformel», cfr. N. LuHmann, Das Recht der Gesellschaft, op. cit., pp. 218-238.

${ }_{24}$ Cfr. C. Rossano, L'eguaglianza giuridica nell'ordinamento costituzionale, op. cit., p. 228. Cfr. N. Luhmann, «Der Gleichheitssatz als Form und als Norm», Archiv für Rechtsund Sozialphilosophie, 77 (1991), pp. 435-445. 
sostituisce l'astrattezza del soggetto. In altre parole, si tratta della sostituzione dell'eteroreferenza (referenze ambientali) con l'autoreferenza (referenze sistemiche) delle operazioni sociali come principio strutturale della formazione dei sistemi. Una astrattezza che consente una inclusione in linea di principio universale, in quanto universale ed astratta è l'individualità. Questa astrattezza trova la sua espressione migliore nel riconoscimento del soggetto giuridico uguale delle codificazioni moderne. L'uguaglianza giuridica è, potremmo dire, presupposto e conseguenza dell'inclusione universale di individui (soggetti) uguali. In questo modo la famiglia non costituisce più il criterio di attribuzione delle individualità, il principio di articolazione della differenziazione dei sottosistemi e della inclusione dei singoli nella società. Ciò non si traduce in una perdita di importanza della famiglia nella modernità. Anzi, la famiglia assume una funzione assolutamente peculiare proprio in virtù di questa astrazione della individualità. Se, infatti l'inclusione nei sistemi di funzione della società moderna (diritto, politica, educazione, economia ecc.) si «normalizza» come inclusione universale, che, in linea di principio, è possibile per tutti gli individui, ciò implica che l'individualità non viene più riconosciuta attraverso riferimenti concreti a questi sistemi, diventando, si potrebbe dire, un fenomeno extra-sociale. Il sistema sociale della famiglia rappresenta un ambito speciale della comunicazione in grado di tematizzare questa forma di individualità $^{25}$. La sua funzione è quella della piena inclusione dell'individuo come persona, il luogo in cui l'astrattezza del riferimento al soggetto trova la concretezza dell'orientamento esclusivo alla singolarità della persona.

Possiamo descrivere uno spostamento, nell'ambito delle riflessioni teoriche, verso una considerazione individualistica della società, insieme alla tesi della separazione dello stato dalla società e la distinzione tra diritto pubblico e diritto privato. L'individuo troverà riconoscimento giuridico nelle moderne codificazioni civili attraverso il soggetto di diritto unico. Le concomitanti codificazioni del diritto pubblico celebrano invece, con il riconoscimento dei diritti fondamentali, la tutela dei diritti politici e civi-

${ }^{25}$ Cfr. R. STichweH, «Systemtheorie der Exklusion. Zum Konflikt von Wohlfahrtsstaatlichkeit und Globalisierung der Funktionssysteme», in ÍD., Die Weltgesellschaft, Frankfurt am Main, Suhrkamp, 2000, pp. 85-102 (89-90). Solo la famiglia e nessun altro sistema può includere l'intera persona dei partecipanti, nel senso che al suo interno la comunicazione può e deve tematizzare ogni azione ed esperienza dei singoli partecipanti, tutto ciò, si potrebbe dire, che non è potenzialmente rilevante, ma anche ciò che è rilevante in altri contesti, in quanto riguarda i singoli partecipanti. Ciò non significa che l'esclusione non sia possibile, solo che essa non è più regolata centralmente, ma «gestita» da criteri specifici per ogni sistema. Cfr. N. Luhmann, Die Gesellschaft der Gesellschaft, vol. II, Frankfurt am Main, Suhrkamp, 1998. 
li del singolo nei confronti del potere dello stato, che porterà a considerare lo stato in funzione del cittadino, del singolo individuo ${ }^{26}$. Si tratta, come abbiamo visto, di una fase di transizione, dove la tesi della separazione dello stato dalla società rappresenta la reazione semantica al passaggio da una società ordinata per strati alla società moderna e dove crescente individualismo si accompagna a crescente differenziazione sociale. Per l'individuo, si tratta della dissoluzione di vincoli particolari, familiari ad esempio, e della partecipazione contemporanea a ruoli differenziati: familiari, professionali, politici, associativi. Non solo cittadino, l'uomo deve «im Wirtschaftssystem mitproduzieren, Kultur überliefern, in zablreichen unpolitischen öffentlichen Angelegenheiten mitwirken, ein Familienleben fübren und dies alles obne zu zerfallen, obne von widerstreitenden Verhaltenspflichten zerrissen zu werden ${ }^{27}$. Ruoli molteplici contemporanei e differenziati, dunque, dove le relazioni sociali si specificano e si differenziano corrispondentemente a nessi materiali e impersonali diversi per ogni contesto. Per questo, riprendendo quanto precedentemente affermato, la comunicazione dell'intimità come forma tipica della comunicazione familiare ha una rilevanza del tutto particolare, proprio in quanto costituisce l'unico contesto della società moderna in cui i contatti si orientano a nessi personali. In questo modo, individualismo della persona e differenziazione sociale possono convivere. Ai diritti fondamentali spetta allora la funzione di garantire questa convivenza tutelando la personalità individuale. L'onere che grava sul comportamento è tale da richiedere una istituzione sociale che assicuri l'individualità dell'individuo a fronte dell'affermazione e del mantenimento di una strutturale sociale funzionalmente differenziata ${ }^{28}$. In questo contesto acquista rilevanza la dimensione affettiva e familiare come unico ambito comunicativo, non residuale, ma necessario, di relazioni sociali personali, ed ha senso la questione posta da Luhmann relativamente alla mancanza di un diritto fondamentale al matrimonio d'amore.

\section{LA FAMIGLIA, IL MATRIMONIO E L'AMORE}

Pur nella mancanza di un diritto fondamentale al matrimonio d'amore, la istituzionalizzazione dell'amore come fondamento del matrimo-

${ }^{26}$ Cfr. G. TARELlo, Storia della cultura giuridica moderna. Assolutismo e codificazione del diritto, Bologna, Il Mulino, 1976, e P. P. Portinaro, Stato, Bologna, Il Mulino, 1999.

${ }_{27}$ Cfr. N. Luhmann, Grundrechte als Institution, op. cit., citazione a p. 48.

${ }^{28}$ Cfr. ivi, pp. 21-22, 50-52. 
nio, abbiamo visto, è un carattere della società moderna che rappresenta un processo di affrancamento del matrimonio da controlli istituzionali e sociali e una forma di rivendicazione di libertà e uguaglianza. Dello stesso segno anche l'autonomizzazione della sfera economica dalla casa: «Non più unità produttiva, la famiglia sembrava diventata puro luogo degli affetti e dei processi di socializzazione primaria [...] e secondaria [...], secondo la fortunata e influente definizione parsonsiana» ${ }^{29}$. Anche il mutamento dei rapporti affettivi e di potere tra i coniugi e tra questi ed i figli rientrano fra quelle trasformazioni che sono all'origine della nascita della famiglia moderna nei paesi occidentali ${ }^{30}$.

Liberazione del matrimonio da vincoli personali e sociali, individualizzazione della persona, molteplicità dei ruoli e differenziazione più marcata tra relazioni impersonali, che aumentano, e relazioni personali, che si fanno più profonde ${ }^{31}$. In questo quadro, ciò che è stata definita come la «perdita di funzioni della famiglia» diventa possibile in quanto la struttura sociale non richiede, e in buona parte non consente più di riconnettere i vincoli creati con il matrimonio (le famiglie) a sostegno di funzioni politiche, religiose o economiche, ma lascia che si connettano in modo del tutto accidentale: per amore, ad esempio. Allo stesso tempo, ciò riformula la rilevanza del matrimonio anche nel senso di un orientamento all'orizzonte futuro del nucleo familiare e non più al passato costituito dalla famiglia di origine, quindi come rifondazione della famiglia ad ogni generazione e non più come perpetuazione o consolidamento delle famiglie (dinastie). A noi interessa qui rilevare come questo, se non poteva garantire la durata dell'amore con il matrimonio, o spiegare i matrimoni infelici, si traduca tuttavia, grazie anche ad una ingerenza sempre minore di fattori religiosi, morali, giuridici e politici, in una crescente tolleranza per i rapporti sessuali prematrimoniali, in una maggiore accettabilità sociale del divorzio e nel livellamento delle differenze dei ruoli sessuali ${ }^{32}$.

A distanza di tempo, forse l'ottimismo di Parsons e Bales relativo alla transitorietà della crisi della famiglia americana non appare giustificato. Il cambio delle funzioni della famiglia non è probabilmente qualcosa che possa strutturalmente risolversi come «riposizionamento funzionale». Questo in quanto è l'individualità a diventare rilevante in seno alla famiglia. L'affettività diffusa che sostiene la comunicazione intima nelle famiglie si orien-

29 C. Saraceno, Sociologia della famiglia, op. cit., p. 179.

${ }^{30}$ M. Barbagli, op. cit., pp. 246-248.

31 N. Luhmann, Sistemi sociali, op. cit., p. 368.

32 Cfr. N. Luhmann, Amore come passione, Roma-Bari, Laterza, 1985 pp. 177-184. 
ta alla specificità individuale dei suoi membri. Anche per questo la storia familiare è così importante nella comunicazione familiare. I ruoli di ciascuno all'interno della famiglia dipendono dalle singole persone, dal loro carattere, e dalla condivisione delle esperienze. In riferimento a ciò, allora, l'intimità presuppone una crescente differenziazione tra rapporti personali e impersonali. Allo stesso tempo, questi rapporti sono sempre più plausibilmente basati sulla uguaglianza dei sessi, mentre, ma non necessariamente in contraddizione, all'interno delle famiglie la rilevanza della individualità accentua la rivendicazione delle differenze, probabilmente anche quelle di genere, ma poi: quale sarebbe l'alternativa per le coppie omosessuali? La peculiarità della famiglia, dove si svolge la piena rappresentazione delle individualità dei suoi membri, configura una nuova crisi, che è propria del carattere affettivo delle relazioni familiari. L'autonomizzazione funzionale della famiglia e dunque dei rapporti intimi, pone il problema della loro stabilità: essa non può essere assicurata dall'esterno, ma è affidata unicamente a risorse personali. Su queste si basa la possibilità del consenso, dell'accordo reciproco, della stabilità, di risolvere i conflitti: può bastare solo l'amore?

Appare chiaro, lo ribadiamo, che la piena inclusione della persona nel sistema famiglia è indipendente dal riconoscimento giuridico del vincolo. E poiché questo tipo di inclusione può avvenire esclusivamente in questo sistema, allora si può affermare che il sistema famiglia tratta come normale un comportamento comunicativo deviante rispetto agli altri siste$\mathrm{mi}^{33}$. Al tempo stesso si rileva come proprio il riconoscimento della individualità, proprio la stessa piena inclusione della persona costituisca un potenziale fattore di conflitto e che l'amore non rappresenti una garanzia di comprensione reciproca. Inoltre, questa inclusione non costituisce più il presupposto per ulteriori inclusioni in altri sistemi di funzione, né può sostituirle. E questo non solo per la natura particolare della comunicazione affettiva intima. Infatti, con una molteplicità di codici distinti, con competenza universale e forma specifica, questo è possibile in quanto i codici binari dispongono di meccanismi di blocco che escludono l'utilizzo di distinzioni che non siano prodotte dal sistema di funzione di volta in volta coinvolto ${ }^{34}$. In questo modo, la società moderna «incoraggia l'assun-

33 Cfr. N. Luhmann, «Sozialsystem Familie», op. cit. La famiglia traccia un confine tra comunicazione intima e comunicazione non intima. Anche se non tutta la comunicazione familiare è comunicazione intima, e quindi non tutta la comunicazione che si svolge in famiglia è codificata dall'amore.

${ }^{34}$ R. Stichweн, «Systemtheorie der Exklusion. Zum Konflikt von Wohlfahrtsstaatlichkeit und Globalisierung der Funktionssysteme», op. cit., pp. 89-90. 
zione di rischi all'interno dei sistemi di funzione. Essa abbatte allo stesso tempo le sicurezze, che prima stavano soprattutto nelle famiglie e quindi nelle strutture della stratificazione, e ne lascia le conseguenze ad un'evoluzione che non può essere controllata centralmente» ${ }^{35}$. Ci chiediamo, tuttavia, cosa questo significhi per la famiglia, a parte il fatto che l'inclusione in questo sistema non sia più indispensabile per le inclusioni negli altri sottosistemi della società, cosa succede con le decisioni, il codice, i programmi, le organizzazioni?

\section{DIRITTO E POLITICA: LA RIFORMA E LA COSTITUZIONE}

Alla luce dei problemi di adeguamento della legislazione ai mutamenti dei costumi e della recezione delle istanze sociali, in relazione ai nostri interessi, sono da segnalare alcune singolarità dell'ordinamento italiano rispetto ad altri paesi. Pur non interessandoci direttamente la questione del matrimonio, i risvolti del testo costituzionale italiano a tutela della famiglia fondata sul matrimonio sono utili alla nostra riflessione in merito alla uguaglianza di genere. La prima riguarda l'art. 29 della Costituzione che riconosce «i diritti della famiglia come società naturale fondata sul matrimonio», senza specificare il sesso dei coniugi, diversamente da altri paesi europei che invece prevedevano nei rispettivi testi costituzionali la eterosessualità dei coniugi. $\mathrm{Ma}$, da una parte è chiaro cosa «intendesse» il costituente italiano, e dall'altra le difficoltà della più rigida formulazione degli ordinamenti stranieri non ha certo impedito a questi ultimi di equiparare le coppie omosessuali a quelle eterosessuali e di prevedere la possibilità di contrarre matrimonio per queste ultime. La seconda è costituita dalla riforma del diritto di famiglia del 1975. Quest'ultima, da un lato, abbiamo visto, rappresenta un tardivo adeguamento al dettato costituzionale, in particolare, al disposto dell'art. 3 nel caso del divieto di discriminazione di genere, e che si sostanzia nell'abbandono del modello familiare gerarchico. Nel suo complesso la riforma si ispira ad ordinamenti più avanzati e tuttavia, se «consideriamo, da un lato, le relazioni intrafamiliari sotto il profilo soggettivo ed affettivo, è ragionevole affermare che siano stati sottolineati valori come quelli della corresponsabilità, dell'intimità, della solidarietà, riferibili al modello della cosiddetta "famiglia coniugale intima" $[. .$.$] Se però, dall'altro lato, guardiamo alla disciplina dei rappor-$

\footnotetext{
35 N. Luhmann, Sociologia del rischio, Milano, Mondadori, 1996, p. 97.
} 
ti patrimoniali, è difficile negare che la riforma si sia ispirata a un modello familiare fondato sulla divisione dei ruoli domestici ed extradomestici sulla base del sesso, con evidenti affinità con l'idealtipo della famiglia parsonsiano» ${ }^{36}$.

L'orientamento normativo complessivo, visto alla luce di quanto è avvenuto nei paesi occidentali dagli anni settanta in poi, è verso quella che è stata definita come degiuridicizzazione e privatizzazione della legislazione sulla famiglia. Piuttosto che un modello normativo preciso delle relazioni familiari, la legislazione si è trasformata in modo da favorire l'autonomia dei singoli componenti della famiglia. Ciò configurerebbe una ridotta volontà di intervento regolativo ed una maggiore flessibilità della legislazione sulla famiglia, dove tutela e controllo anziché sulla famiglia si orienterebbero agli individui ${ }^{37}$. Due aspetti ulteriori meritano di esser sottolineati: una sostanziale sovrapposizione tra la deistituzionalizzazione espressa in questa tendenza legislativa e quella che emerge come scelta di allontanamento dall'istituzionalizzazione del rapporto di coppia, come dimostrato dall'aumento delle coppie che optano, ad esempio, per la convivenza al di fuori del matrimonio. Fatto, quest'ultimo, che si suppone dovuto anche alla volontà di evitare l'intervento residuale dello stato nei casi di crisi della coppia. Al tempo stesso, tuttavia, e siamo al secondo aspetto, a fronte di questo allontanamento dall'istituzione matrimoniale, si registra negli ultimi anni un fenomeno in controtendenza, relativo alle richieste di regolazione giuridica delle convivenze e delle coppie omosessuali. Richieste che hanno sollecitato interventi legislativi in tal senso, con risultati molto differenti nei vari paesi, e che animano tuttora il dibattito etico e politico ${ }^{38}$.

D'altra parte, se colleghiamo le considerazioni relative al cambiamento delle modalità di controllo e tutela istituzionale della famiglia con le considerazioni relative alla sua funzione, è possibile affermare che la scelta legislativa di orientarsi ai singoli componenti, più che alla famiglia nel suo complesso, è in linea con le forme di inclusione della società moderna ed anche con la funzione di inclusione delle persone, della specificità della loro individualità, dei ruoli caratteriali, svolta dalla famiglia. Con questo,

36 V. POCAR e P. RONFANI, «Le trasformazioni della famiglia italiana e l'intervento della legge e del giudice», in ÍD., Forme, op. cit., pp. 101-116, 106. Cfr. M. BARBAGLI, op. cit., p. 99.

37 P. RONFANI, «Dalla convivenza prematrimomiale al matrimonio informale», in V. Pocar e P. Ronfani, Coniugi senza matrimonio, Milano, Raffaello Cortina, 1992, pp. 3-47 (24-25).

${ }^{38}$ Ivi, p. 25. Cfr. V. POCAR e P. RONFAnI, «Le trasformazioni della famiglia italiana e l'intervento della legge e del giudice», op. cit., pp. 114-115. 
la rivendicazione delle differenze diventa una richiesta non solo legittima, ma anche necessaria alla piena partecipazione alla famiglia, fatto salvo un accordo sostanziale sulla conduzione della vita in comune. In ogni caso, è la stessa natura della comunicazione intima che pretende riconoscimento per la individualità. Dello stesso segno le rivendicazioni di unioni familiari non sancite dal diritto, oppure di riconoscimento giuridico per i «matrimoni informali», per le coppie omosessuali, per le adozioni ecc. Forme non previste dall'ordinamento, che però irritano ancor più altri ambiti già sollecitati dal tema della famiglia «naturale» e del matrimonio.

Il riferimento alla famiglia, infatti, giustifica atteggiamenti fortemente antitetici con le stesse motivazioni. Basti osservare il dibattito sul riconoscimento delle unioni di fatto e sull'ammissibilità dei matrimoni omosessuali. Ci si può chiedere, a questo proposito, quanto la legislazione relativa alla famiglia abbia tenuto conto della realtà dei comportamenti concreti, accogliendo modelli alternativi a quello istituzionalizzato della famiglia tradizionale. Anche se ciò non sembra sia avvenuto, tuttavia, l'intera disciplina è stata formulata in modo da non assumere modelli alternativi rigidamente delineati, ma tale da risultare aperta e adattabile alla molteplicità delle forme dei comportamenti concreti. Ne è seguito, da un lato, una tendenza sostanzialmente invariata nella diminuita propensione al matrimonio per le coppie eterosessuali, dall'altro l'aumento delle richieste di riconoscimento giuridico per le coppie omosessuali, come anche delle opposizioni a queste ultime. Si assiste allora a tendenze di senso opposto, verso la privatizzazione e la deistituzionalizzazione, in un caso, verso la regolamentazione giuridica e il riconoscimento istituzionale dall'altro.

\section{UGUAGLIANZA, STRUTTURA E TEMPO}

La modernità ha fatto della realizzazione dell'uguaglianza un obiettivo politico. Ci chiediamo non solo se questo sia un obiettivo realizzabile, ma anche se l'uguaglianza sia in sé espressione di un ordine, o comunque fornisca un criterio ordinatore. Apparentemente non basta l'uguaglianza, quanto meno non basta l'affermazione dell'uguaglianza per l'instaurazione di un ordine. Questo è quanto è stato affermato a proposito della distruzione dell'ordine antico avvenuta con l'affermazione della società moderna. Il passato si espone alla osservazione ed alla critica ed il futuro diventa la dimensione temporale privilegiata, sulla quale proiettare il mutamento, verso una direzione da decidere: «und das, was in der Gesellschaft als 
natürlich und notwendig gilt, wird in dieser Perspektive etwas Artifizielles und Kontingentes. Aber daraus folgt nicht, daß mann auch sagen könnte, wie es anders zu machen wäre» ${ }^{39}$. Anche i fondatori della sociologia sono consapevoli che con la distruzione dell'ordine precedente, la Rivoluzione non abbia però proposto un ordine alternativo ${ }^{40}$. Su questo si articola tutto il discorso dell''llluminismo e della modernità che è alla base della nascita della sociologia come scienza, come evidenzia, ad esempio, Habermas: «L'oggetto della sociologia (la società) nasce soltanto a partire da una certa prospettiva teorica. Ad esempio la problematica filosofica del giusrazionalismo - come si possa realizzare, con strumenti di diritto positivo, un'associazione di cittadini liberi ed eguali- dischiude un orizzonte emancipativo di aspettative proprio con l'indirizzare lo sguardo sulle resistenze di una realtà sociale che sembra irragionevole» ${ }^{41}$.

L'enfasi si proietta in questo modo sull'orizzonte temporale, sia nel senso che la distruzione dell'ordine precedente sottrae autorità alla tradizione nella giustificazione di un presente che non può più essere dato per scontato e proprio per questo, nell'altro senso, istituzionalizza il mutamento, spostando l'accento sulla dimensione del futuro ${ }^{42}$. Questo spostamento si collega storicamente alla nascita della società borghese e concettualmente alla ideologia dell'uguaglianza. Qui l'uguaglianza ha una funzione peculiare rispetto alla dimensione temporale: «The bürgerliche Gesellschaft has been a revolutionary society with a strong structural emphasis on time and corresponding simplifications of social and environmental relations. The principle of its future was simply the denial of its past by the antistructural postulate of equality» ${ }^{43}$. Qui troviamo insieme due negazioni compiute dal postulato di uguaglianza: la negazione del passato e la negazione della stessa idea di principio strutturale (ordine). Le due negazio-

39 N. Luhmann, Die Gesellschaft der Gesellschaft, op. cit., p. 1119.

${ }^{40}$ Cfr., ad esempio, S. Landshut, «Zum Begriff und Gegenstand der Politischen Soziologie», Kölner Zeitschrift für Soziologie und Sozialpsychologie, 8 (1956), pp. 410-414.

${ }^{41}$ J. Habermas, La costellazione postnazionale, Milano, Feltrinelli, 2002, p. 30. Così, continua Habermas citando Landshut: «"Società" è soltanto l'etichetta attribuita all'insieme delle tensioni, contraddizioni e problemi derivanti dalla ricaduta delle idee di libertà ed eguaglianza» (ivi).

${ }_{42}$ Cfr., tra gli altri, A. Borghini, Metamorfosi del potere, Milano, Franco Angeli, 2003.

${ }^{43}$ N. Luhmann, «The Future Cannot Begin: Temporal Structures in Modern Society», Social Research, 1 (1976), pp. 130-152 (151), che aggiunge in nota: «Since equality implies freedom and freedom implies inequality, the postulate of equality cannot refer to reality, but only to time. Its only function is to deny the relevance of the past - e.g., the relevance of biographies and ascribed status for the access to education (equality of opportunity) or to political elections» (ivi). 
ni strutturano ampi spazi di possibilità per soluzioni alternative, contingenze appunto, che si proiettano verso il futuro. La costituzione, afferma Luhmann, «deve rimpiazzare quei sostegni esterni che erano stati postulati dal giusnaturalismo» ${ }^{44}$. Al tempo stesso funge da programma operativo per il diritto, come insieme di regole per individuare casi e risolverli mediante decisione, sottolinea Corsi. Per far ciò occorrono, per così dire, da un lato, i valori e, dall'altro le organizzazioni con i loro procedimenti. Organizzazioni che consentono di combinare la legislazione con la giurisdizione, la politica con il diritto, la programmazione di scopo, orientata al futuro, con la programmazione condizionale indifferente al futuro, mentre la costituzione fornisce loro le premesse decisionali. Così, per poter esser tradotti in decisioni i valori devono poter essere specificati nei procedimenti, proprio a partire dalla loro inconsistenza semantica: «è qui che diventa indispensabile la vacuità semantica dei valori e dei principi: il principio di uguaglianza, per fare l'esempio più eclatante, è accettato universalmente solo perché non specifica affatto i criteri della sua applicazione, oppure, detto altrimenti, perché non dà alcun elemento per essere riconosciuto. Ciò viene lasciato all'apparato organizzativo e solo il procedimento può costruire argomentazioni per decidere se le differenze riscontrate sono compatibili con il principio di uguaglianza» ${ }^{45}$. Così, le differenze, che prima erano naturali e costituivano un dato ex ante, diventano qui artificiali, nel senso che sono costruite attraverso i procedimenti, ex post potremmo dire, ma avendo come dato di partenza lo spazio indifferenziato aperto dai valori. Tutto ciò è naturalmente possibile grazie alla forma della costituzione che consente l'accoppiamento strutturale tra politica e diritto e che contiene diritti fondamentali (valori) e regole procedurali ${ }^{46}$.

A livello semantico, inoltre, i valori possono esser descritti come un «unviolate level of order», che non viene messo in discussione dalle contingenze, né è disturbato, ma riaffermato da paradossi e tautologie insiti nelle

${ }^{44}$ N. LuHMAnN, «La costituzione come acquisizione evolutiva», in G. ZAGREBELSKY, P. Portinaro e J. Luther (a cura di), Il futuro della costituzione, Torino, Einaudi, 1996, pp. 83-128 (94).

${ }^{45}$ G. CORSI, «Sociologia da Constituição», Revista da Faculdade de Direito - Universidad Federal de Minas Gerais, 39 (2002), pp. 169-189.

46 N. LuHmanN, «La costituzione come acquisizione evolutiva», op. cit., sottolinea come «l'accoppiamento strutturale è una forma particolare nel senso di Spencer Brown, cioè una differenziazione a due lati. Dal lato interno, si facilita l'influenza reciproca che viene "legalizzata" dalla costituzione. Dal lato esterno, tale influenza viene per quanto possibile esclusa, o quantomeno resa illegale. Politica e amministrazione del diritto sono tenute a rapportarsi l'una all'altra "solo in modo conforme alla costituzione" e non altrimenti» (p. 113). 
autodescrizioni della società: «values are "blind spots" that make it possible for systems to observe and act» ${ }^{47}$.

\section{DIRITTI UGUALI PER SOGGETTI UGUALI?}

D'altra parte, evidenzia Giancarlo Corsi, lo svuotamento non riguarda solo il contenuto di quei diritti ma anche i loro destinatari, come espressi dai termini «soggetto», «persona», «individuo»: anche qui la universalizzazione dei diritti viene pagata con la mancanza di referenti concreti ${ }^{48}$. Il soggetto di diritto è una costruzione giuridica che, astraendo dalle connotazioni concrete dei soggetti, consente di avere un destinatario unico per le sue norme ${ }^{49}$. Nonostante l'insistenza, dopo la costituzionalizzazione dei diritti fondamentali, sulla universalità dei diritti, si fa fatica a sostenerne il riconoscimento al di fuori delle categorie dello stato nazionale e del possesso di una soggettività giuridica. Come se il riconoscimento sociale del soggetto si esprimesse pienamente solo con la sua definizione giuridica. L'astrazione necessaria all'affermazione dell'uguaglianza sulle differenze che il diritto riconosceva in base a referenze concrete ha come contraltare lo svuotamento semantico, abbiamo visto, rispetto al contenuto, ma anche in relazione ai destinatari concreti. La figura dei diritti soggettivi, alla quale in ultima istanza sono riconducibili i diritti fondamentali, ha avuto in questo un ruolo fondamentale, rendendo disponibile un orientamento altamente astratto per il diritto e l'agire. Il riferimento al valore, sottolinea De Gior-

${ }^{47}$ Così N. Luhmann, «Tautology and Paradox in the Self-Descriptions of Modern Society», in ÍD., Essays on Self-Reference, New York, Columbia University Press, 1990, pp. 123-143 (133-134). Luhmann inserisce il discorso sui valori nell'ambito dei problemi di self-reference relativi alle autodescrizioni della società, problemi che vengono affrontati grazie ad espedienti semantici appunto, i quali nascondono l'indescrivibilità della descrizione, ma permettono l'autodescrizione. Così per i concetti di «stato»e «individuo» usati in opposizione alla società, secondo una «alibi function» che non viene svelata dalla ideologizzazione e temporalizzazione dei concetti, ma che la rende precaria. Rispetto a ciò, conclude Luhmann, «values provide the corresponding explanation: new "inviolate levels" are required if everything appears to be contingen and if communication itself must test what will work as a starting point for the unfolding of self-reference» (ivi, pp. 137-138).

${ }^{48}$ Così. G. CoRsi, «Valores y derechos fundamentales en perspectiva sociológica», Metapolitica, 5 (2001), pp. 159-169.

${ }_{49}$ Ma il soggetto ha anche una funzione per le analisi della sociologia: «Il soggetto è una figura del discorso. Se questa figura non funziona, ci si deve chiedere quale problema avesse risolto e occultato». Cfr. N. LUHMANN, «Modernità e differenziazione sociale», in G. MARI (a cura di), Moderno postmoderno. Soggetto, tempo, sapere nella società attuale, Milano, Feltrinelli, 1988, pp. 88-97. 
gi, «relativizza tanto il diritto quanto la soggettività. Il soggetto di diritto e il diritto soggettivo sostituiscono il risultato stabile del riferimento reciproco di due forme contingenti di valore: da una parte il valore d'ordine proprio del diritto e dall'altra il valore in sé della soggettività individuale» ${ }^{50}$. Diventa dunque necessario colmare questo vuoto, dare concretezza alle astrazioni, ma in mancanza di criteri di riferimento. La figura del cittadino non è stata sufficiente. Così, accanto alle dichiarazioni universali dei diritti (umani), i «diritti speciali» sono tentativi di fissare referenze concrete nello «spazio dell'astrazione». Sfilano categorie speciali di ogni tipo, ce n'è per tutti, soprattutto categorie formate in base a differenze naturali: l'età, il sesso, le (diverse) capacità. Le donne, dunque, accanto a bambini, anziani, diversamente abili ${ }^{51}$. Con ciò si delinea un cambiamento fondamentale nelle forme dell'inclusione sociale e quindi nel perseguimento dell'uguaglianza e nella giustificazione della disuguaglianza.

Qui si può vedere come con la modernità la questione si ponga decisamente nei termini della differenza uguaglianza/disuguaglianza. E sappiamo anche che una parte della forma non può esistere senza l'altra. La garanzia dell'inclusione universale, dell'uguale accesso ai sottosistemi sociali non elimina, ma sposta solo in avanti il momento della produzione della disuguaglianza. L'inclusione non rende uguali, ma rende possibile attivare differenze sulla base di rilevanze che sono specifiche di ogni sistema di funzione. Così, è proprio lo svuotamento ad essere il presupposto che permette a questi diritti inseriti nella costituzione di svolgere la loro funzione nella società moderna: i valori aprono uno spazio dove è possibile specificare determinate forme ${ }^{52}$.

50 R. De Giorgi, «Semantica dell'idea di diritto soggettivo», in N. Auciello e R. RaciNARO (a cura di), Storia dei concetti e semantica storica, Napoli, Esi, 1990, pp. 119-130 (126): «Lo stato sociale dispone, attraverso la figura giuridica della soggettività, di uno strumento insieme di specificazione e di universalizzazione. La soggettività diventa una finzione operativa che permette di tenere costantemente aperta la catena di condizionalità necessaria alla realizzazione di programmi politici» (ivi, p. 127). Cfr. N. LuHMANN, «Diritti soggettivi. Mutamenti della coscienza giuridica per la società moderna», Sociologia e politiche sociali, 1 (2001), pp. 10-59.

${ }^{51} \mathrm{Ma}$ anche le minoranze di ogni tipo, come le culture, i popoli indigeni, e altri, che, a differenza dei diritti fondamentali individuali, sono espressione di una reazione al problema dell'esclusione sociale. Cfr. G. ConSI, «Inclusione. La società osserva l'individuo», Teoria sociologica, 1 (1993), pp. 279-301 (p. 281, nota 2), dove viene citato un passo tratto da T. Parsons, The Evolution of Societies, Englwood Cliffs, Prentice-Hall, 1977, pp. 189-190, che qui riporto: «Departures from the egalitarian principle must be justified, either on the basis of incapacity to participate fully —as among small children - or of being qualified for special contributors, as through competence, to the societal welfare».

52 G. CoRsI, «Sociologia da Constituição», op. cit. Non sono, sottolinea Corsi, diretta- 
Ma come si riflette l'evoluzione delle forme di articolazione della vita familiare e della rappresentazione sociale dell'intimità nella trasformazione del diritto di famiglia? Le trasformazioni più recenti del diritto di famiglia negli ordinamenti europei, operate sulla base della evidenza dei mutamenti sociali avvenuti nei diversi paesi e della necessità di un crescente riconoscimento dei diritti individuali, si sono risolte in una riduzione delle differenze esistenti tra gli ordinamenti dei vari paesi, in virtù della sostanziale similarietà dei mutamenti sociali. Così, si delinea la tendenza verso un'attribuzione delle differenze ancora esistenti in termini di legislazione sulla famiglia a fattori dipendenti dalla differente capacità dei diversi governi (in termini geografici e cronologici) di rispondere alle richieste di riforma, a fronte di una accresciuta enfasi sui valori comuni ${ }^{53}$.

\section{FAMIGLIE SENZA MODELLI}

Il problema della mancanza di un diritto fondamentale al matrimonio d'amore si pone nell'attualità in forza delle richieste di unioni tra individui dello stesso sesso. Ciò in quanto, per questi ultimi, la possibilità di contrarre matrimonio non appartiene storicamente alle acquisizioni delle nostre legislazioni che invece da sempre riconoscono il matrimonio tra individui di sesso diverso. Allora, rispetto alle coppie eterosessuali, per le quali, in numero sempre maggiore, l'esercizio della libertà di scelta del partner e delle forme di organizzazione della vita in comune è una scelta che si esprime al di fuori delle istituzioni, una rivendicazione in questo senso da parte dei movimenti omosessuali non potrà che essere un'opzione per una «giuridificazione dell'affettività», poiché da sempre esclusi da questo riconoscimento. Senza porre la questione della irrilevanza del genere sessuale in merito alla dimensione dell'affettività, qui diventano invece rilevanti le possibilità di altre inclusioni proprio in quanto, potremmo dire, l'astrattezza del soggetto non funziona, non garantisce l'inclusione ${ }^{54}$.

mente le organizzazioni a determinare le disuguaglianze, ma a queste viene affidata la produzione delle differenze.

53 Così, L. BALestra, «L'evoluzione del diritto di famiglia e le molteplici realtà affettive», in T. Auletta (a cura di), Famiglia e matrimonio, Torino, Giappichelli, 2010, pp. 1-27 (1). A questo proposito, l'autore evidenzia come si sia parlato di «convergenze tecniche» e «convergenze naturali» nella riduzione delle differenze tra gli ordinamenti (ivi).

${ }_{54}$ Per una rilfessione sui diritti all'orientamento sessuale come caso particolare della tutela della privacy, cfr. R. PRANDINI e M. BorTolini, «Il diritto alla privacy come diritto 
Tentiamo qui di vedere se e come siano cambiate effettivamente le cose per le famiglie dopo la riforma e le trasformazioni più recenti del diritto di famiglia. Da un certo punto di vista, si potrebbe formulare la questione in questo modo: la riforma del diritto di famiglia ha raggiunto i suoi obiettivi? Teniamo presente che in qualche modo, in continuità con il passato, il diritto continua ad essere uno strumento di riconoscimento e consolidamento dell'ordine sociale. Questo è evidente anche quando guardiamo al soggetto astratto, socialmente de-qualificato, del diritto che finisce per essere, da qui l'universalità della sua figura, la base di legittimazione dell'ordine sociale. Così il panorama dei comportamenti sociali e delle norme giuridiche relativi alle relazioni familiari anteriormente alla riforma mostrano un quadro composito. Da una parte la famiglia appare omologa alla società, ne riproduce i rapporti di potere ed i ruoli distinti per genere. Il diritto riconosce dunque questo tipo di dinamiche, garantendo, al tempo stesso, i diritti dei singoli componenti e l'autorità del pater familias giustificata sulla base della dipendenza economica della famiglia. Ed ancora: la libertà di scelta del partner è garantita, ma insieme al potere patriarcale. In questo modo, si delinea una situazione apparentemente singolare, dove le norme costituzionali, ci riferiamo qui soprattutto all'art. 3 e all'art. 29, più giovani di pochi anni rispetto al codice del 1942, sono di tutt'altro segno rispetto al diritto di famiglia dell'epoca e, dunque, ai concreti rapporti del tempo. Ci si richiama, infatti, a una eguaglianza tra uomini e donne che non ha riscontro nei rapporti sociali, né, abbiamo detto, nelle norme che regolano la famiglia. D'altra parte, occorre dire, anche per la costituzione, come per il codice, la sola famiglia ammessa sembra essere quella tradizionale, dunque quella legittima fondata sul matrimonio, al cui interno la sessualità appare regolata, anche sulla base della subordinazione femminile. D'altra parte, è stata denunciata a questo proposito una certa «ipocrisia di fondo» da parte del legislatore, il quale, se non è riuscito a disciplinare il fenomeno in maniera organica, ha però nei fatti equiparato la posizione del convivente more uxorio a quella del coniuge in relazione ad interessi specifici ${ }^{55}$.

In generale, a proposito dei nuovi modelli familiari proposti dal codice, è possibile evidenziare come questi siano ispirati a una forte democra-

soggettivo. Semantica della neutralizzazione e struttura della società», Sociologia e politiche sociali, 1 (2001), pp. 74-132.

55 Cfr. L. BALESTRA, «L'evoluzione del diritto di famiglia e le molteplici realtà affettive», op. cit., p. 6: «Ciò a riprova di come sia sufficiente che l'attenzione venga distolta dal tema nella sua portata complessiva per consentire alla politica di procedere senza veti di matrice ideologica» (ivi). 
tizzazione dei rapporti. Con l'adeguamento alla previsione costituzionale e al mutamento dei costumi e degli stili di vita, la riforma del diritto di famiglia del 1975 in Italia si presenta con una marcata innovazione rispetto a quei tempi, stabilendo la parità dei coniugi e la sostanziale de-gerarchizzazione delle relazioni. Così, in relazione ai rapporti tra realtà sociale, costituzione e codice civile, è stato notato come le differenze tra una costituzione ispirata all'uguaglianza e un codice civile che struttura gerarchicamente le relazioni uomo-donna si dovrebbero alla prospetticità del documento costituzionale, che contiene una prospettiva ideologica, un programma di sviluppo sociale e non era quindi rispondente alla realtà pre-riforma, ben rappresentata invece nel codice previgente. Con la riforma si raggiungerebbe allora il risultato del pieno coordinamento tra costituzione, codice e realtà sociale. Il punto che ci interessa sottolineare, rispetto a questo rapporto, è che oggi la realtà sembra aver superato la portata o la comune interpretazione dei precetti costituzionali e le regole della riforma ${ }^{56}$, aprendo uno scenario per certi versi inedito per quel panorama normativo e che costringe a riflettere proprio sulla questione dell'uguaglianza di genere e sui ruoli interni alla famiglia.

Intanto sono da rivedere anche le posizioni dei sociologi relativamente alla funzione della famiglia e del matrimonio. Non solo la posizione parsonsiana, ma anche quella, parzialmente sovrapponibile, di Luhmann nel suo scritto del 1965. La famiglia, infatti, diventa soprattutto l'ambito dello sviluppo e del consolidamento della comunicazione affettiva intima. Così, una volta venuta meno la base stabile del patriarcato e i ruoli di genere distinti, le relazioni intime si basano solo sull'affettività, e dunque sono anarchiche, transitorie. Non sorprende dunque la scelta sempre più diffusa di creare una famiglia al di fuori del matrimonio. Ciò che sorprende è invece l'idea che possano bastare divieti, imposizioni o, se del caso, minacce di scomunica a rendere duratura l'unione. In ogni caso, procreazione, continuità generazionale, trasmissione del patrimonio, ad esempio, non sono più caratteri fondamentali dell'unione familiare. Gli effetti della riforma, tuttavia, sembrano bloccarsi di fronte all'art. 29 della Costituzione, in particolare alla sua interpretazione nel senso del riconoscimen-

${ }^{56}$ Cfr. R. TommasinI, «I rapporti personali tra coniugi», in T. AuletTA (a cura di), Famiglia e matrimonio, Torino, Giappichelli, 2010, pp. 424-482 (424-427): «È significativo che i precetti costituzionali - pure di rango superiore - per moltissimi anni non abbiano inciso sulle norme del codice, ancora regolatrici della materia. Era il segno che la società, ed anche la società familiare, non era pronta a recepire il contenuto dei precetti costituzionali» (p. 425). 
to della famiglia fondata sul matrimonio come unico modello «naturale», lasciando fuori dalla protezione costituzionale modelli alternativi a quello tradizionale e ormai anche più diffusi di quello. Adesso è il codice che sembra più moderno della norma costituzionale. Per fortuna, si affermano interpretazioni differenti per le quali la famiglia non sarebbe portatrice di interessi sovraordinati a quelli dei singoli componenti. Si può descrivere in questo modo il risultato raggiunto nei termini del riconoscimento della centralità dei valori della persona, di una sorta di individualizzazione degli interessi dei singoli soggetti coinvolti, di una attenzione prioritaria allo sviluppo delle personalità individuali nel rispetto di valori costituzionali legati all'uguaglianza, alla democrazia, e alle libertà soggettive ${ }^{57}$. Ciò vuol dire contestualizzare la famiglia nel momento storico della società contemporanea e del suo diritto, nel senso che i rapporti familiari «non si possono sottrarre — data l'unitarietà dell'ordinamento (corsivo mio) — ad un giudizio di meritevolezza, ad un confronto con i valori fondamentali del sistema giuridico vigente, con il suo ordine pubblico» ${ }^{58}$. D'altra parte, una società che mostra come «il modello della famiglia centrata sulle relazioni affettive di natura interpersonale sarà destinato ad evolversi, a partire dagli anni Ottanta, verso quello della cosiddetta famiglia post-moderna d'impronta individualistica, nella quale, sulle aspirazioni alla felicità di coppia, tenderanno a prevalere le aspirazioni alla felicità dei singoli individui nella coppia» ${ }^{59}$. Al tempo stesso è da sottolineare come, «nel sistema del 1975 è certamente presente la consapevolezza di una nuova fisionomia della famiglia, che è diventata luogo di ricerca dell'accordo e quindi di svolgimento dell'autonomia negoziale, per cui è stata recuperata nell'ambito familiare la categoria dell'autonomia privata e del negozio giuridico, largamente discussa e contestata per la regolamentazione dei rapporti personali» ${ }^{60}$.

Così, quindi, le unioni non basate sul matrimonio non sarebbero giuridicamente irrilevanti, fatto salvo il rispetto dei valori suddetti. Si tratta quindi di riconoscere e dare rilievo alle motivazioni di una scelta fatta in difformità rispetto al matrimonio come base dell'unione. Questa scelta è dunque espressione dell'autonomia individuale, che rispetta i valori dell'ordinamento in quanto non mette in discussione la rilevanza del matrimonio, ma solo rivendica la sua non esclusività. In questo modo diven-

${ }^{57}$ Cfr. V. Pocar e P. Ronfani, La famiglia e il diritto, op. cit., pp. 33-35.

${ }^{58}$ Cfr. P. Perlingieri, La persona e $i$ suoi diritti, Napoli, ESI, 2005, pp. 369-375.

59 Così, V. Pocar e P. Ronfani, La famiglia e il diritto, citato in L. BALESTRA, «L'evoluzione del diritto di famiglia e le molteplici realtà affettive», op. cit., p. 10, nota 37 .

${ }^{60}$ R. Tommasini, «I rapporti personali tra coniugi», op. cit., p. 431. 
ta possibile differenziare le scelte, senza riconoscere come giuridicamente irrilevanti quelle che, prescindendo dal matrimonio, manifestano comunque una volontà di sottrarsi alla istituzionalizzazione. È possibile quindi rispettare la scelta in difformità, senza escluderla; considerarla giuridicamente rilevante, rispettandone la volontà di non essere completamente «assorbita» nell'alveo del modello rispetto al quale quella scelta ha inteso differenziarsi. La rilevanza giuridica di queste scelte, e quindi la loro inclusione nel sistema, va dunque graduata. Questa impostazione assorbirebbe anche le convivenze omosessuali, una volta che siano stati riconosciuti loro contenuto e funzioni atipiche ${ }^{61}$.

\section{MATRIMONI DI GENERE?}

Diversa è la questione, ancora più radicale, del matrimonio tra persone dello stesso sesso. Intanto, però è da osservare come all'estero vi sia già stata una equiparazione, in molti casi per via legislativa, della coppia omosessuale a quella eterosessuale ed il dibattito si è spostato sull'ammissibilità del matrimonio omosessuale. Non così in Italia, dove parte della dottrina è molto cauta sul tema e tendenzialmente contraria ad ogni equiparazione ${ }^{62}$.

In ogni caso, la legalizzazione del matrimonio omosessuale riapre la questione relativa al riconoscimento del matrimonio come diritto civile (fondamentale, umano, universale). Nell'ambito dell'evoluzione storica di quei diritti (soggettivi), il riconoscimento di diritti sociali alla famiglia sta ad indicare un diverso rapporto tra stato e società. Questi diritti, d'altra parte, sono riferibili all'unione già in essere e non al suo momento costitutivo. A ben vedere, con riguardo a questo momento, sembra possibile descrivere nel corso del tempo un processo di generalizzazione del diritto e del soggetto giuridico che interessa anche il matrimonio, dove le differenze di genere restano le sole differenze naturali ammesse, o potremmo dire: pretese. La regolamentazione giuridica dell'unione è una regolamentazione dell'atto che costituisce l'unione e deve riferirsi alla differenza di genere, indicare il maschio e la femmina. Le regolamentazioni si riferiscono quindi al momento costitutivo: sono esterne al matrimonio in quanto ne sono alla base ${ }^{63}$. Il senso

${ }^{61}$ Cfr. ivi, pp. 483-498.

${ }^{62}$ Così, L. Balestra, «L'evoluzione del diritto di famiglia e le molteplici realtà affettive», op. cit., pp. 16-18.

${ }_{63}$ Vi sono, è vero, regolazioni in tema di diritto successorio, di separazione e di divorzio, ma sono tutte forme che si riferiscono alla dissoluzione della coppia. Sulla capacità di 
della regolazione giuridica dello spazio familiare, è questo il nostro ragionamento, può e deve essere rintracciato nell'adeguamento giuridico alle differenze rilevanti, quelle naturali, ma anche etniche o religiose, ammesse e dunque, in quanto tali anche razionali. La loro irrilevanza rende, da una parte, il senso della generalizzazione del soggetto e richiede, dall'altra, una specificazione dell'individuo da parte del diritto, una sorta di rimaterializzazione dei soggetti e dei contenuti. Le formulazioni normative, infatti, devono contenere specificazioni relative alle possibili scelte individuali ed agli status (convivenze al di fuori del matrimonio, unioni civili, patti di convivenza, matrimoni tra persone dello stesso sesso), dove anche l'individuo non è considerato astrattamente, ma è invece biologicamente e socialmente connotato.

Non sembra diversamente conciliabile la costituzionalizzazione di un diritto alla scelta delle relazioni intime con una iperindividualizzazione dei soggetti e la richiesta che le scelte individuali ricevano garanzie di tipo giuridico. In realtà, soprattutto nel dibattito italiano pur nell'assenza di un preciso modello di unione familiari, che in ogni caso non corrisponderebbe alla realtà sociale, permangono distinzioni, laddove, ad esempio si continua a considerare la famiglia tradizionale il «valore» (reale o fittizio) di riferimento per un riconoscimento surrogato (anche a livello normativo) di forme alternative a tale modello. Esistono dati culturalmente presupposti, e fra questi: il matrimonio tra persone di sesso differente come base della famiglia; e non invece: la libera scelta delle relazioni affettive. Anche il dato costituzionale non può prescinderne, nonostante la modernità degli assunti e tantomeno le dichiarazioni dei diritti, quando omettono di indicare la differenza di genere come base del matrimonio, in quanto lo danno per scontato. Persino, a maggior ragione, la Chiesa quando si esprime sul riconoscimento dei diritti umani non fa riferimento al matrimonio come base dell'unione familiare. Questa omissione, d'altra parte, non ha impedito al diritto, di fronte all'emersione e alla sempre maggiore rilevanza di modelli alternativi alla famiglia tradizionale, di resistere ai fatti. Tuttavia, non è più certamente sostenibile una naturalità ed eticità delle unioni tradizionali. Nè tantomeno la irrazionalità di forme ad essa alternative.

intervento del diritto sulle dinamiche relazionali della coppia, legate alla dissoluzione del rapporto, alle sue patologie, sembra illuminante anche qui ciò che Perlingieri rilevava a proposito della tutela dei diritti del minore, relativamente alla formazione ed alla sensibilità dei giudici minorili, spesso provenienti dalle sezioni penali e fallimentari: «Il fallimento della famiglia è ben altra cosa»; oppure, con una rilevanza ancora più estesa, in relazione agli avvocati: «l'avvocato della famiglia è il divorzista, il separazionista, quello che vive sul fallimento della famiglia, sul momento patologico della famiglia». Cfr. P. PerLingieri, La persona e $i$ suoi diritti, op. cit., p. 304. 
In ogni caso, forse è possibile tentare una prima risposta alle questioni di partenza, tenendo presente i processi attivati dall'innovazione rappresentata dall'idea di diritto soggettivo, dove si possono osservare crescenti differenziazioni delle identità personali. Tali identità «esistono ormai solo come finzioni operative nella totale inclusione nel sistema del diritto. E allora $[\ldots]$ si può senz'altro affermare che il presente $[\ldots]$ sta non più nella pretesa riaffermazione di una identità priva di sostanza, ma piuttosto nella legittima aspettativa di un diritto alla differenza» ${ }^{64}$.

\section{DONNE E UOMINI}

Interpretazioni del principio di uguaglianza nel senso di trattare ciò che è uguale in modo uguale e ciò che è disuguale in modo disuguale, nelle loro varie versioni, sono una tautologia normativa, concludeva Podlech, «die es unter Hinzufügung weiterer Prämissen [...] oder ibres Gegenteils gestattet, von jedem beliebigen sozialen Zustand zu erklären, daß er in Übereinstimmung mit ibm stehe» ${ }^{65}$. Fra queste premesse che darebbero senso a quelle interpretazioni, a titolo esemplificativo, Podlech inserisce «Frauen haben Männern untertan zu sein» («le donne devono essere sottomesse agli uomini», ed è proprio quello che prevedeva il codice del 1942 in Italia) oppure il contrario. Pensiamo che correttivi di questa interpretazione come l'equità o la ragionevolezza delle distinzioni non abbiano altro risultato se non quello di spostare in avanti il momento della differenza di trattamento. Il senso della impossibilità dell'uguaglianza è solo occultato da formule più o meno raffinate. La questione dell'uguaglianza tra uomo e donna è paradigmatico. Non è necessaria una trattazione separata, tutto ciò che è stato detto finora in relazione all'uguaglianza vale anche per l'uguaglianza di genere. Qui è però possibile vedere meglio come, anziché riconoscere la differenza «naturale» tra uomo e donna come presupposto per un trattamento differenziale, questa differenza venga negata a favore della loro uguaglianza, presupposta come naturale, per poi essere reintrodotta successivamente, ma con una argomentazione del tipo: uomini e donne sono diversi, ma noi dobbiamo trattarli come uguali e a tal fine dobbiamo differenziarli ma non in base alla loro evidente diversità ${ }^{66}$. Così,

${ }^{64}$ Così, R. De Giorgi, «Semantica dell'idea di diritto soggettivo», op. cit., pp. 129-130.

${ }^{65}$ A. PODLECH, Gebalt und Funktionen des allgemeinen verfassungsrechtlichen Gleichbeitssatzes, op. cit., p. 57.

${ }_{66}$ Perché, si chiede De Giorgi, «la scienza giuridica ha abbandonato lo schema dei dirit- 
infatti, Podlech sottolinea come il comma II dell'art. 3 della Costituzione tedesca non vada inteso nel senso della necessità di un trattamento eguale tra uomo e donna, bensì come proibizione di un trattamento diseguale che abbia a motivo la differenza tra uomo e donna. A queste condizioni, continua riferendosi al Bundesverfassungsgericht, «Die Begründung mit den "biologischen und funktionalen" Eigenarten der Frau ist jedoch geeignet die Interpretation des art. 3 abs. 2 und 3 GG zu verunsichern» ${ }^{67}$. Proprio ciò che evidentemente è differente, deve essere negato. Appunto quelle differenze, che sono genuinamente naturali, negate da una uguaglianza che si presume naturale. Ma neanche il loro riconoscimento servirebbe di per sé ad una concreta applicabilità se non è utile a costruire asimmetrie. Perciò più sopra si faceva riferimento ad una regola aggiuntiva del tipo «le donne devono essere sottomesse all'uomo» o al suo contrario «gli uomini devono essere sottomessi alle donne». Questo sì che fornisce indicazioni operative. In fondo, non lasciava adito a dubbi il diritto di famiglia, quando le posizioni erano differenziate, ma ancora, si badi bene, non grazie alle diversità biologiche ecc., ma sulla base della supposizione della superiorità del ruolo maschile. Lo abbiamo visto, pur sinteticamente ed evitando letture critiche e di genere ${ }^{68}$. La cosa, è naturale, non ci vede d'accordo sulla superiorità dell'uno sull'altra; ciò che ci interessa è rilevare la presenza di un'asimmetria ${ }^{69}$. Così, quando si giunge a «riformare» in direzione di un adeguamento tardivo al dettato costituzionale e, si dice, al mutamento dei costu$\mathrm{mi}$, non sorprende il fatto che la riforma si risolva in un modello aperto e flessibile, come è stato definito, mentre mantiene asimmetrie in relazione

ti naturali per passare dalla natura, dalla ragione al soggetto, e quindi all'individuo? E per costruire poi il sistema dei diritti soggettivi intesi come proprietà, come attributi del soggetto, $\mathrm{i}$ quali gli spetterebbero in quanto uomo e quindi, ancora una volta, per natura, per la sua natura umana? Perché, in altri termini, si è escluso il principio della natura per reimmetterlo alla fine, togliendolo dall'inizio? Perché, in altri termini, introdurre nel sistema un paradosso e lavorare poi continuamente alla sua deparadossizzazione o tautologizzazione (come si vuole dire)?». Cfr. R. De GIORGI, «Semantica dell'idea di diritto soggettivo», op. cit., p. 125.

${ }^{67}$ A. Podlech, Gehalt und Funktionen des allgemeinen verfassungsrechtlichen Gleichbeitssatzes, op. cit., pp. 91-92 (nota 8).

${ }^{68}$ Cfr. N. Luhmann, «Frauen, Männer und Georg Spencer Brown», Zeitscbrift für Soziologie, 1 (1988), pp. 47-71, e A. BARATTA, «Il progetto giuridico della differenza e il mito dell'unità», in R. DE GIORgI (a cura di), Il diritto e la differenza. Studi in onore di Alessandro Baratta, vol. 1, Lecce, Pensa Multimedia, 2002, pp. XXIX-XXXIX.

69 Sembra che sbilanciamenti estremi non possano essere evitati, soprattutto quando si pensa di dover «compensare» antichi squilibri che pesano sul presente: si veda il caso delle c.d. «azioni positive». In Spagna, ma non solo, per la custodia dei figli in caso di separazione veniva fortemente privilegiata la madre; situazione sanata di recente a favore della «custodia compartida». 
agli aspetti patrimoniali. E d'altra parte, cos'altro ci si potrebbe aspettare dal linguaggio giuridico? Ci si richiama proprio alla logica dell'accordo e dell'autonomia negoziale per definire l'apertura del nuovo diritto di famiglia rispetto alla regolamentazione dei rapporti personali.

Per concludere, forse è un'ultima riflessione sulla famiglia che può suggerire una risposta. Forse è proprio nella costruzione della sua autonomia, nella costruzione del riferimento ricorsivo alla osservazione di osservazioni, nella mancanza di organizzazioni e programmi, scopi e condizioni e nell'orientamento ai racconti. Le necessarie differenziazioni dei ruoli nella quotidianità, che non avvengono più sulla base di una precostituita differenza/gerarchia tra $i$ generi, non è più una questione di genere, ma può anche esserlo. Il diritto dà prova della impossibilità di regolare la questione. L'adeguamento alla costituzione in direzione della uguaglianza si risolve in una flessibilità della legislazione: su questa base si dovrà poi decidere caso per caso. Ma come decidere su ciò che la famiglia fa quotidianamente con la costruzione della sua storia, della storia del sistema? «Es mag dann durchaus, feministisch angeregt, um die Frage gehen, ob nicht heute einmal der Mann das Geschirr abwaschen oder wenigstens beim Abtrocknen belfen sollte. Aber zu einen Kommunikation des Familiensystem wird dies nur im Kontext eines Rückgriff auf die Frage, wer gestern, wer vorgestern, wer bisher immer abgewaschen hat; oder darauf, wie gerade vorher das Gespräch bei Tisch verlaufen ist; oder auch im Kontext mit anderen "Tests" dieser Art; oder in der Erwartung, daß man sich daraufbin leichter entschließen wird, eine Geschirrspülmaschine zu kaufen; und alles in allem: durch die Beobachtung der damit verbundenen Beobachtungen ${ }^{70}$.

${ }^{70}$ N. Luhmann, «Sozialsystem Familie», op. cit., p. 206. 